\title{
Extension of the 5-alkynyluridine side chain via C-C-bond formation in modified organometallic nucleosides using the Nicholas reaction
}

\author{
Renata Kaczmarek ${ }^{1}$, Dariusz Korczyński ${ }^{1}$, James R. Green ${ }^{\star 2}$ and Roman Dembinski ${ }^{* 1,3}$
}

\section{Letter}

Address:

${ }^{1}$ Department of Bioorganic Chemistry, Centre of Molecular and Macromolecular Studies, Polish Academy of Sciences, Sienkiewicza 112, 90-363 Łódź, Poland, ${ }^{2}$ Department of Chemistry and Biochemistry, University of Windsor, Windsor, Ontario, N9B 3P4, Canada, and ${ }^{3}$ Department of Chemistry, Oakland University, 146 Library Drive, Rochester, Michigan 48309-4479, USA

Email:

James R. Green* - jgreen@uwindsor.ca; Roman Dembinski* dembinsk@oakland.edu

* Corresponding author

Keywords:

alkynes; 5-alkynyluridines; C-C-bond formation; dicobalt hexacarbonyl complexes; Nicholas reaction; nucleosides; propargyl cation
Beilstein J. Org. Chem. 2020, 16, 1-8. doi:10.3762/bjoc.16.1

Received: 05 September 2019

Accepted: 14 November 2019

Published: 02 January 2020

Associate Editor: S. Flitsch

(c) 2020 Kaczmarek et al.; licensee Beilstein-Institut. License and terms: see end of document.

\begin{abstract}
Dicobalt hexacarbonyl nucleoside complexes of propargyl ether or esters of 5-substituted uridines react with diverse C-nucleophiles. Synthetic outcomes confirmed that the Nicholas reaction can be carried out in a nucleoside presence, leading to a divergent synthesis of novel metallo-nucleosides enriched with alkene, arene, arylketo, and heterocyclic functions, in the deoxy and ribo series.
\end{abstract}

\section{Introduction}

Nucleoside analogs are molecules of high pharmacological interest for the treatment of various conditions, especially cancer and viral diseases [1-5]. The substitution at C-5 of the uracil nucleobase provides a common framework for materials with potent biological properties [6-10]. Modification on this site of the nucleobase usually does not interfere with Watson-Crick base pairing. For example, C-5-modified pyrimidines are well tolerated by commercial polymerases $[11,12]$ Alkynyl modifications not only provide a biological impact but also create a synthetic handle for further functionalization/modification. Among others, alkynyl uridines undergo cycloisomerization to potent antiviral agents, furopyrimidines [13], related halofuropyrimidines [14], and can be converted into interstrand dimers [15].

In parallel, bioorganometallic chemistry provides new tools to influence biological interactions [16-24]. Cobalt possesses a diverse array of properties that can be manipulated to yield 
promising drug candidates [25]. The antiproliferative properties [26], as well as carbon monoxide-releasing properties $[27,28]$ of dicobalt hexacarbonyl alkyne complexes have been noted, and their medicinal potential has been summarized [29$31]$.

Despite developments, the collection of metallo-nucleosides is limited. Hybridization of alkyl and aryl-substituted alkyne cobalt hexacarbonyls with 2'-deoxyuridines revealed pronounced in vitro activity against MCF-7 and MDA-MB-231 human breast cancer cells [32,33]. A recent investigation of hexacarbonyl dicobalt adducts of nucleosides containing derivatives of propargyl alcohol demonstrated their antiproliferative activities for the HeLa and K562 cell lines [31]. The formation of a reactive oxygen species in the presence of cobalt compounds was determined in K562 cells. The results indicate that the mechanism of action for most antiproliferative cobalt compounds may be related to the induction of oxidative stress [31]. Consequently, we aimed to develop methods that would synthetically extend the design of the metallo-nucleosides by introducing functionalized ligands in divergent synthesis. We decided to pursue the Nicholas reaction in the presence of the labile nucleoside unit, further modifying the already available material containing the propargyl alcohol derivative unit.

The chemistry of cationic propargyl dicobalt complexes, recognized as the Nicholas reaction, has become one of the most widely appreciated forms of metalorganic chemistry. These cations are generated most commonly from propargyl alcohol, -ether, or -acetate hexacarbonyl dicobalt complexes and a Lewis or Brønsted acid. A range of heteroatom nucleophiles have been incorporated into alkyne dicobalt complexes by this chemistry [34-40]. However, reactions with carbon-based nucleophiles provide an opportunity to access the structurally diverse products via formation of $\mathrm{C}-\mathrm{C}$ bonds. Nucleophiles as diverse as electron-rich arenes or heteroarenes [41,42], alkenes [43], allylmetalloids [44-46], enol derivatives [47,48], and organometallics [49] are suitable for the Nicholas reaction. Allenic byproducts are rarely seen, and intramolecular versions of the reaction are also highly successful [50,51].

Although the Nicholas reaction has been employed to functionalize biomolecules, including amino acids [52,53], $\beta$-lactams [54], steroids [55], and carbohydrates [56-62], we are unaware of any examples of nucleoside functionalization by way of propargyl dicobalt cation chemistry. Nucleoside modifications are considerably challenging due to the presence of reactive functional groups. Since numerous uridine C-5 modifications play an important role in biochemistry, we considered exploration of pertinent methods development warranted, which at the same time may provide biologically active compounds.

\section{Results and Discussion}

Preparation of 5-alkynyluridines was carried out from acyl-protected 5-iodouridines (1a,b) $[8,63]$ and the appropriate terminal alkyne in the presence of catalytic amounts of $\mathrm{Pd}\left(\mathrm{PPh}_{3}\right)_{4}$, copper(I) iodide, triethylamine, in DMF, and at room temperature - to avoid cycloisomerization to furopyrimidines (Scheme 1). The modified pyrimidine nucleoside scaffolds, propargyl acetate-substituted 2'-deoxyuridine ( $\mathrm{R}=\mathrm{Ac}, 2)$ and propargyl methyl ether-substituted uridine $(\mathrm{R}=\mathrm{Me}, \mathbf{3})$, were obtained in $87 \%$ and $61 \%$ yield, respectively. These specific combinations were not optimized since we presumed that acetate and methyl ether could be used interchangeably. Acetyl protection has been introduced to alcohol functions to prevent free hydroxy groups from competing with the C-nucleophiles in

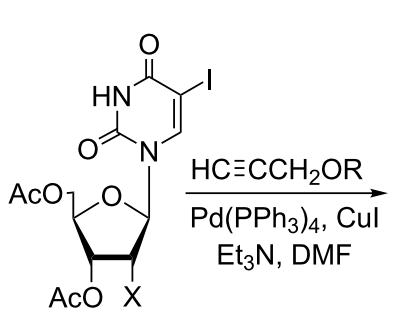

1a, $X=H$

1b, $X=O A C$

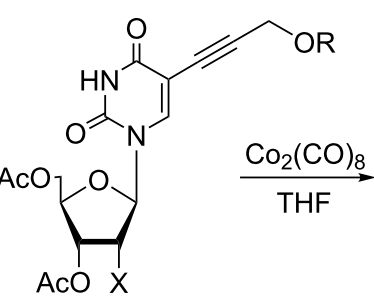

2, $\mathrm{R}=\mathrm{Ac}, \mathrm{X}=\mathrm{H}$

3, $\mathrm{R}=\mathrm{Me}, \mathrm{X}=\mathrm{OAc}$

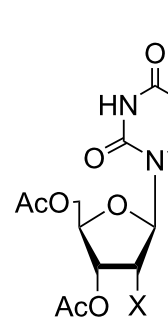

4, $\mathrm{R}=\mathrm{Ac}, \mathrm{X}=\mathrm{H}$ 5, $\mathrm{R}=\mathrm{Me}, \mathrm{X}=\mathrm{OAC}$

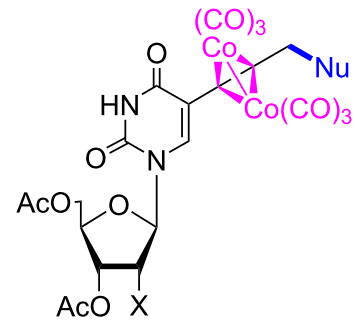

6, $\mathrm{R}=\mathrm{X}=\mathrm{H}$

7, $\mathrm{R}=\mathrm{X}=\mathrm{OAC}$

$\mathrm{Nu}-\mathrm{Y}=$

a) $\mathrm{Me}_{3} \mathrm{SiCH}_{2} \mathrm{CH}=\mathrm{CH}_{2}$

b) 1,3,5-trimethoxybenzene

c) acetophenone trimethylsilyl enol ether

d) N-methylindole 
the Nicholas reaction. The structures of alkynyl nucleosides 2 and 3 were confirmed by ${ }^{1} \mathrm{H}$ and ${ }^{13} \mathrm{C}$ NMR spectroscopy and high-resolution mass spectrometry $[64,65]$.

The conversion of alkynyl nucleosides $\mathbf{2}$ and $\mathbf{3}$ into the corresponding dicobalt hexacarbonyl nucleosides complexes of $\mathbf{4}$ and 5 was accomplished at room temperature $\left(\mathrm{Co}_{2}(\mathrm{CO})_{8}\right.$, THF $22{ }^{\circ} \mathrm{C}, 1 \mathrm{~h}$ ) with $88-77 \%$ yield after silica gel column chromatography (Scheme 1). The structures of nucleosides $\mathbf{4}$ and $\mathbf{5}$ were confirmed by NMR and IR spectroscopy (for the synthesis of a related unprotected nucleoside, see [31]). The MS spectra of $\mathbf{4}$ and $\mathbf{5}$ exhibited appropriate high resolution molecular ions' masses.

The solutions of uridine complexes $\mathbf{4}$ and $\mathbf{5}$ in dichloromethane were subjected to Nicholas reactions with a variety of diverse nucleophiles in the presence of $\mathrm{BF}_{3} \cdot \mathrm{OEt}_{2}$. Representatives of the major classes of $\mathrm{C}$-based nucleophiles in Nicholas reaction chemistry were selected, including electron-rich arenes, $\pi$-excessive heterocycles, enol derivatives, and allylmetalloids. Specifically, the reactivity of 1,3,5-trimethoxybenzene, $N$-methylindole, acetophenone trimethylsilyl enol ether, and allyltrimethylsilane was investigated (Table 1). The Nicholas reaction products $\mathbf{6}$ and $\mathbf{7}$ (Figure 1) were obtained successfully in moderate to good yields (Table 1). The reactions progressed quite slowly and required an excess amount of the Lewis acid (4-5 equiv) to proceed at a preparatively reasonable rate (Table 1, entries 1-4). These observations can be attributed to the substantial number of potentially competing Lewis basic sites in $\mathbf{4}$ and $\mathbf{5}$. The use of tin(IV) chloride (stannic chloride) provided generally a slightly faster reaction but with slightly lower yields, except in the case of the $\mathbf{5}$ /allyltrimethylsilane/7a combination (Table 1, entry 5). Reactions were more successful when the amount of nucleophile present was in slight excess relative to that of the Lewis acid, whereas limited amounts of nucleophile resulted in greater amounts of decomposition. Slightly more decomposition products were observed by TLC in reactions with ribo nucleoside 5 (Table 1, entries 4-7) than with 2'-deoxy derivative 4 (Table 1 , entries $1-3$ ), leading to higher yields for nucleosides $\mathbf{6 a - c}$ relative to $\mathbf{7 a}, \mathbf{b}, \mathbf{d}$ (Table 1).
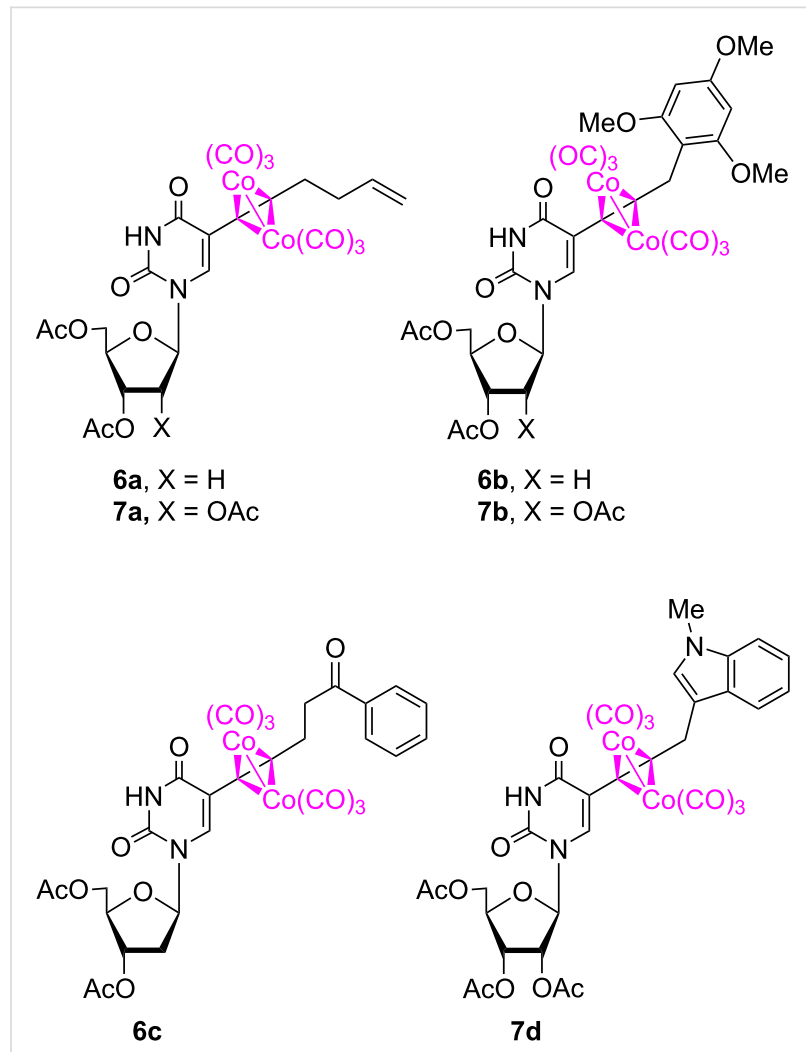

Figure 1: Structures of nucleosides 6 and 7 , products of the Nicholas reaction.

The reaction products were characterized by the disappearance of the formally diastereotopic propargylic methylene ${ }^{1} \mathrm{H}$ NMR $\left(\mathrm{CDCl}_{3}\right)$ spectral resonances (ca. $5.5 \mathrm{ppm}$ in $\mathbf{4}$ and $4.8 \mathrm{ppm}$ in 5) and their reappearance upfield in the reaction products (i.e., $3.17 \mathrm{ppm}$ in 6a and $4.27 \mathrm{ppm}$ in $\mathbf{6 b}$ ). In the ${ }^{13} \mathrm{C}$ NMR spectra, the slightly broadened resonance of the metal carbonyl carbons

\begin{tabular}{|c|c|c|c|c|}
\hline entry & nucleoside & nucleophile & product & yield [\%] \\
\hline 1 & 4 & allyltrimethylsilane & $6 a$ & 55 \\
\hline 2 & 4 & 1,3,5-trimethoxybenzene & $6 b$ & 89 \\
\hline 3 & 4 & acetophenone trimethylsilyl enol ether & $6 c$ & 49 \\
\hline 4 & 5 & allyltrimethylsilane & $7 a$ & 38 \\
\hline 5 & 5 & allyltrimethylsilane & $7 a$ & $37(46)^{a, b}$ \\
\hline 6 & 5 & 1,3,5-trimethoxybenzene & $7 b$ & 47 \\
\hline 7 & 5 & $N$-methylindole & $7 d$ & 40 \\
\hline
\end{tabular}

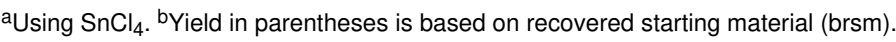


at 199-200 ppm (199.4 ppm in both $6 \mathbf{a}$ and $\mathbf{6 b})$ were characteristic of the product alkyne- $\mathrm{Co}_{2}(\mathrm{CO})_{6}$ complexes. In the IR spectra, the series of intense metal-CO stretching bands between 2000-2100 cm $\mathrm{cm}^{-1}$ (e.g., 6a, 2089, 2049, $2017 \mathrm{~cm}^{-1}$; $\mathbf{6 b}$, 2088, 2048, $2018 \mathrm{~cm}^{-1}$ ) dominated even the organic carbonyl bands.

\section{Conclusion}

The Nicholas reaction, in which site of reactivity is welldefined and commonly free from formation of allenic byproducts, has been introduced into the repertoire of nucleosides modifications. The reaction of dicobalt hexacarbonyl propargylic alcohol uridine derivatives has been validated with diverse C-nucleophiles. By this means, alkene, arene, arylketo, and heterocyclic functions can be introduced onto metallo-nucleosides, preserving the dicobalt hexacarbonyl unit. This methodology allows for access in a divergent fashion to a variety of modified nucleosides with potential biological activity, and was shown to be viable for both 2'-deoxy- and regular uridines.

\section{Experimental}

General and instrumentation. All NMR measurements were carried out on Bruker Avance III spectrometers operating for ${ }^{1} \mathrm{H}$ NMR at $500 \mathrm{MHz}, 600 \mathrm{MHz}$ or $300 \mathrm{MHz}$ and for ${ }^{13} \mathrm{C} \mathrm{NMR}$ at $125 \mathrm{MHz}$ or $150 \mathrm{MHz}$, at $22^{\circ} \mathrm{C}$. Mass spectra were recorded on an Agilent 6520 Q-TOF LCMS (HRMS). FTIR spectra were recorded on ATI Mattson Infinity Series AR60, Thermo Scientific Nicolet 6700 ATR, or Bruker Alpha-P ATR spectrometers. All reactions were carried out under a nitrogen atmosphere and all products were stored in a freezer at $-10^{\circ} \mathrm{C}$.

3',5'-Di-O-acetyl-2' -deoxy-5-[3-(acetoxy)prop-1-yn-1yl]uridine (2). A round-bottom flask was charged with 3',5'-di$O$-acetyl-2'-deoxy-5-iodouridine (1a, $0.500 \mathrm{~g}, 1.14 \mathrm{mmol})$, $\mathrm{Pd}\left(\mathrm{PPh}_{3}\right)_{4}(0.066 \mathrm{~g}, 0.057 \mathrm{mmol}), \mathrm{CuI}(0.011 \mathrm{~g}, 0.057 \mathrm{mmol})$, DMF $(10 \mathrm{~mL}), \mathrm{Et}_{3} \mathrm{~N}(396 \mu \mathrm{L}, 2.85 \mathrm{mmol})$, and propargyl acetate $(283 \mu \mathrm{L}, 2.85 \mathrm{mmol})$. The reaction mixture was stirred at room temperature for $22 \mathrm{~h}$. The solvent was removed by oil pump vacuum, and the residue was purified using silica gel column chromatography (230-400 mesh, eluent: $0 \rightarrow 2 \%$ methanol in chloroform). The product was dried by oil pump vacuum for $2 \mathrm{~h}$ to give 2 as a white foam $(0.405 \mathrm{~g}, 0.992 \mathrm{mmol}, 87 \%)$ ${ }^{1} \mathrm{H}$ NMR (500 MHz, DMSO- $d_{6}$ ) $\delta 11.75$ (s, $\left.1 \mathrm{H}, \mathrm{N}-\mathrm{H}\right), 8.01$ (s, $1 \mathrm{H}, \mathrm{H}-6), 6.12$ (t, $\left.J=6.9 \mathrm{~Hz}, 1 \mathrm{H}, \mathrm{H}-1^{\prime}\right), 5.19-5.15$ (m, 1H, H-3'), 4.87 (s, 2H, $\mathrm{CH}_{2}$ ), 4.27-4.23 (m, 2H, H-4', H-5'), 4.21-4.17 (m, 1H, H-5"), 2.52-2.47 (m, 1H, H-2'), 2.35-2.28 (m, 1H, H-2"), $2.07\left(\mathrm{~s}, 3 \mathrm{H}, \mathrm{CH}_{3}\right), 2.05\left(\mathrm{~s}, 6 \mathrm{H}, 2 \mathrm{CH}_{3}\right)$; ${ }^{13} \mathrm{C}$ NMR $\left(125 \mathrm{MHz}, \mathrm{DMSO}-d_{6}\right) \delta 170.10,170.03,169.71$, $161.30,149.33,144.40,97.85,87.05,84.90,81.48,78.52$, 73.66, 63.53, 52.20, 36.17, 20.76, 20.54, 20.46; IR ( $\left.\mathrm{cm}^{-1}, \mathrm{KBr}\right)$ 3442 m, 3389 m, 2987 m, 2823 m, 1701 s, 1627 s, 1467 m,
$1288 \mathrm{~m}, 1052 \mathrm{~m}$; TOF-ESI ${ }^{+}-\mathrm{MS}(\mathrm{m} / \mathrm{z}):[\mathrm{M}+\mathrm{Na}]^{+}$calcd for $\mathrm{C}_{18} \mathrm{H}_{20} \mathrm{~N}_{2} \mathrm{NaO}_{9}, 431.1061$; found, 431.1064 .

$2^{\prime}, 3^{\prime}, 5^{\prime}$-Tri- $O$-acetyl-5-(3-methoxyprop-1-yn-1-yl)uridine (3). A round-bottom flask was charged with 2 ', 3',5'-tri- $O$ acetyl-5-iodouridine (1b, 0.500g, $1.01 \mathrm{mmol}), \mathrm{Pd}\left(\mathrm{PPh}_{3}\right)_{4}$ (0.058 g, $0.050 \mathrm{mmol})$, CuI (0.010 g, $0.050 \mathrm{mmol})$, DMF $(10 \mathrm{~mL}), \mathrm{Et}_{3} \mathrm{~N}(351 \mu \mathrm{L}, 2.52 \mathrm{mmol})$, and methyl propargyl ether $(212 \mu \mathrm{L}, 2.52 \mathrm{mmol})$. The reaction mixture was stirred at room temperature for $22 \mathrm{~h}$. The solvent was removed by oil pump vacuum, and the residue was purified using silica gel column chromatography (230-400 mesh, eluent: $0 \rightarrow 2 \%$ methanol in chloroform). The product was dried by oil pump vacuum for $2 \mathrm{~h}$ to give 3 as a white foam $(0.270 \mathrm{~g}, 0.616 \mathrm{mmol}, 61 \%)$. ${ }^{1} \mathrm{H}$ NMR $\left(500 \mathrm{MHz}, \mathrm{CDCl}_{3}\right) \delta 8.78(\mathrm{~s}, 1 \mathrm{H}, \mathrm{N}-\mathrm{H}), 7.79(\mathrm{~s}, 1 \mathrm{H}$, H-6), 6.08-6.06 (m, 1H, H-1'), 5.35-5.30 (m, 2H, H-3', H-4'), 4.40-4.37 (m, 1H, H-2'), 4.37-4.35 (m, 2H, H-5', H-5"), 4.28 (s, $\left.2 \mathrm{H}, \mathrm{CH}_{2}\right), 3.40\left(\mathrm{~s}, 3 \mathrm{H}, \mathrm{OCH}_{3}\right), 2.21\left(\mathrm{~s}, 3 \mathrm{H}, \mathrm{CH}_{3}\right), 2.12(\mathrm{~s}, 3 \mathrm{H}$, $\left.\mathrm{CH}_{3}\right), 2.11\left(\mathrm{~s}, 3 \mathrm{H}, \mathrm{CH}_{3}\right) ;{ }^{13} \mathrm{C} \mathrm{NMR}\left(125 \mathrm{MHz} \mathrm{CDCl}_{3}\right) \delta$ $170.13,169.65,169.55,160.68,142.06,100.66,90.35,87.46$, 80.19, 75.44, 73.20, 70.01, 62.91, 60.30, 57.88, 51.08, 20.83, 20.54, 20.45; IR (cm $\left.{ }^{-1}, \mathrm{KBr}\right) 3208$ br w, 3082 br w, 2938 br w, 2823 br w, 1743 s, 1692 vs, 1628 m, 1453 m, 1214 vs, 1092 s; TOF-ESI ${ }^{+}$MS $(m / z):[\mathrm{M}+\mathrm{Na}]^{+}$calcd for $\mathrm{C}_{19} \mathrm{H}_{22} \mathrm{~N}_{2} \mathrm{NaO}_{10}$, 461.1167; found, 461.1171 .

General procedure for the synthesis of hexacarbonyl dicobalt 5-alkynyluridines (4 or 5): A round-bottom flask was charged under a nitrogen atmosphere with $\mathrm{Co}_{2}(\mathrm{CO})_{8}(0.222 \mathrm{~g}, 0.650 \mathrm{mmol})$, alkynyl nucleoside 2 or 3 $(0.500 \mathrm{mmol})$, and THF $(10 \mathrm{~mL})$. The mixture was stirred at room temperature $\left(22^{\circ} \mathrm{C}\right)$ for $1 \mathrm{~h}$. The solvent was removed by rotary evaporation. Silica gel column chromatography (230-400 mesh, eluent: chloroform) gave reddish-brown compounds 4 or 5 .

Hexacarbonyl dicobalt 3',5'-di-O-acetyl-2'-deoxy-5-[3(acetoxy)prop-1-yn-1-yl]uridine (4). From alkynyl nucleoside $2(0.204 \mathrm{~g}, 0.500 \mathrm{mmol})$; brown foam $(0.305 \mathrm{~g}, 0.440 \mathrm{mmol}$, $88 \%) ;{ }^{1} \mathrm{H}$ NMR (600 MHz, $\left.\mathrm{CDCl}_{3}\right) \delta 9.32(\mathrm{~s}, 1 \mathrm{H}, \mathrm{NH}), 7.83$ (s, $1 \mathrm{H}, \mathrm{H}-6), 6.26-6.22$ (m, 1H, H-1'), 5.57-5.47 (m, 2H, $\mathrm{CH}_{2}$ ), 5.23-5.20 (m, 1H, H-3'), 4.41-4.37 (m, 1H, H-4'), 4.32-4.29 (m, 1H, H-5'), 4.28-4.24 (m, 1H, H-5"), 2.66-2.61 (m, 1H, H-2'), 2.17-2.11 (m, 7H, H-2", 2CH $\mathrm{CH}_{3}, 2.07$ (s, 3H, $\mathrm{CH}_{3}$ ); ${ }^{13} \mathrm{C}$ NMR $\left(150 \mathrm{MHz}, \mathrm{CDCl}_{3}\right) \delta 198.71,170.73,170.29,170.26$, $160.23,149.43,138.26,113.71,94.82,85.91,82.58,79.29$, $74.03,65.45,63.65,37.93,20.90,20.60,20.54$; IR ( $\left.\mathrm{cm}^{-1}, \mathrm{KBr}\right)$ 3356 br m, 3089 br w, 2960 br w, 2093 m, 2056 s, 2024 br s, $1736 \mathrm{vs}, 1638 \mathrm{~m}, 1561 \mathrm{~m}, 1406 \mathrm{~m}, 1228 \mathrm{vs}, 1024 \mathrm{~s}$; TOF-ESI ${ }^{+}-\mathrm{MS}(\mathrm{m} / \mathrm{z}):[\mathrm{M}+\mathrm{Na}]^{+} \mathrm{calcd}$ for $\mathrm{C}_{24} \mathrm{H}_{20} \mathrm{Co}_{2} \mathrm{~N}_{2} \mathrm{NaO}_{15}$, 716.9420; found, 716.9426 . 
Hexacarbonyl dicobalt $2^{\prime}, 3^{\prime}, 5^{\prime}$-tri- $O$-acetyl-5-(3methoxyprop-1-yn-1-yl)uridine (5). From alkynyl nucleoside 3 (0.219 g, $0.500 \mathrm{mmol})$; brown foam $(0.279 \mathrm{~g}, 0.385 \mathrm{mmol}$, $77 \%) ;{ }^{1} \mathrm{H}$ NMR $\left(500 \mathrm{MHz}, \mathrm{CDCl}_{3}\right) \delta 9.56(\mathrm{~s}, 1 \mathrm{H}, \mathrm{NH}), 7.74(\mathrm{~s}$, $1 \mathrm{H}, \mathrm{H}-6), 6.10-6.00$ (m, 1H, H-1'), 5.41-5.30 (m, 2H, H-3', H-4'), 4.79 (m, 2H, $\mathrm{CH}_{2}$ ), 4.41-4.27 (m, 3H, H-5', H-5", H-2'), $3.54\left(\mathrm{~s}, 3 \mathrm{H}, \mathrm{OCH}_{3}\right), 2.15\left(\mathrm{~s}, 3 \mathrm{H}, \mathrm{CH}_{3}\right), 2.12\left(\mathrm{~s}, 3 \mathrm{H}, \mathrm{CH}_{3}\right), 2.10$ $\left(\mathrm{s}, 3 \mathrm{H}, \mathrm{CH}_{3}\right) ;{ }^{13} \mathrm{C} \mathrm{NMR}\left(125 \mathrm{MHz}, \mathrm{CDCl}_{3}\right) \delta 198.97,170.42$, 169.66, 160.56, 150.01, 138.54, 114.36, 96.13, 87.92, 79.99, 79.19, 73.53, 72.44, 70.30, 63.34, 59.12, 20.69, 20.59, 20.44; IR $\left(\mathrm{cm}^{-1}, \mathrm{KBr}\right) 3234$ br w, $2991 \mathrm{w}, 2092 \mathrm{~m}, 2051 \mathrm{~s}, 2004$ vs, $1746 \mathrm{~m}, 1688 \mathrm{~m}, 1447 \mathrm{~m}, 1214$ br m, $1094 \mathrm{~m}, 750 \mathrm{vs}$; TOF-ESI ${ }^{+}-\mathrm{MS}(\mathrm{m} / \mathrm{z}):[\mathrm{M}+\mathrm{Na}]^{+}$calcd for $\mathrm{C}_{25} \mathrm{H}_{22} \mathrm{Co}_{2} \mathrm{~N}_{2} \mathrm{NaO}_{16}$, 746.9526; found, 746.9536 .

Hexacarbonyl dicobalt 3',5'-di- $O$-acetyl-2'-deoxy-5-(hex-5en-1-yn-1-yl)uridine (6a). To a solution of nucleoside complex $4(0.0206 \mathrm{~g}, 29.7 \mu \mathrm{mol})$ in $\mathrm{CH}_{2} \mathrm{Cl}_{2}(5 \mathrm{~mL})$ at $0{ }^{\circ} \mathrm{C}$ was added allyltrimethylsilane $(25 \mu \mathrm{L}, 0.16 \mathrm{mmol})$ and $\mathrm{BF}_{3} \cdot \mathrm{OEt}_{2}$ $(15 \mu \mathrm{L}, 0.12 \mathrm{mmol})$. The solution was stirred over $12 \mathrm{~h}$ with gradual warming to room temperature, at which time starting material consumption was complete, as evidenced by TLC (1:1 petroleum ether/EtOAc). Then, $\mathrm{NH}_{4} \mathrm{Cl}$ (saturated aq, $0.25 \mathrm{~mL}$ ) and $\mathrm{NaHCO}_{3}$ (saturated aq, $0.25 \mathrm{~mL}$ ) were added, followed by $\mathrm{MgSO}_{4}$. The mixture was filtered through a plug of silica gel and washed with EtOAc. Concentration of the crude reaction product and purification by flash chromatography $(2: 1 \rightarrow 3: 2$ petroleum ether/EtOAc) afforded $\mathbf{6 a}$ as a red-brown oil (0.0110 g, $16.3 \mu \mathrm{mol}, 55 \%) .{ }^{1} \mathrm{H} \mathrm{NMR}\left(500 \mathrm{MHz}, \mathrm{CDCl}_{3}\right) \delta$ 8.85 (br s, 1H), 7.72 (s, 1H), 6.24 (dd, $J=8.0 \mathrm{~Hz}, 5.4 \mathrm{~Hz}, 1 \mathrm{H})$, $5.93(\mathrm{~m}, 1 \mathrm{H}), 5.22(\mathrm{~d}, J=6.3 \mathrm{~Hz}, 1 \mathrm{H}), 5.15(\mathrm{~d}, J=17.2 \mathrm{~Hz}$, $1 \mathrm{H}), 5.05(\mathrm{~d}, J=10.1 \mathrm{~Hz}, 1 \mathrm{H}), 4.37(\mathrm{dd}, J=11.6,5.1 \mathrm{~Hz}, 1 \mathrm{H})$, 4.30 (br s, $1 \mathrm{H}), 4.25$ (dd, $J=11.6,3.6 \mathrm{~Hz}, 1 \mathrm{H}), 3.17$ (apparent t, $J=7.9 \mathrm{~Hz}, 2 \mathrm{H}), 2.61(\mathrm{dd}, J=14.0,4.6 \mathrm{~Hz}, 1 \mathrm{H}), 2.43(\mathrm{dt}, J=$ 8.1, 7.0 Hz, 2H), 2.13 (s, 3H), 2.12 (obscured, 1H), 2.08 (s, $3 \mathrm{H}) ;{ }^{13} \mathrm{C} \mathrm{NMR}\left(125 \mathrm{MHz}, \mathrm{CDCl}_{3}\right) \delta 199.4,170.3,159.9,149.4$, 137.2, 115.6, 114.6, 103.6, 85.7, 82.4, 81.8, 74.0, 63.7, 37.9, 35.6, 33.8, 20.9, 20.6; IR (neat, ATR) 3197, 3077, 2967, 2089, 2049, 2017, 1747, 1714, 1691, $1587 \mathrm{~cm}^{-1}$; ESI ${ }^{+}-\mathrm{MS}$ $(m / z):[\mathrm{M}]^{+}$calcd for $\mathrm{C}_{25} \mathrm{H}_{22} \mathrm{Co}_{2} \mathrm{~N}_{2} \mathrm{O}_{13}, 698.9684$; found, 698.9689 .

Hexacarbonyl dicobalt 3',5'-di- $O$-acetyl-2'-deoxy-5-[3-(2,4,6trimethoxyphenyl)prop-1-yn-1-yl]uridine (6b). To a solution of nucleoside complex $4(0.0210 \mathrm{~g}, 30.3 \mu \mathrm{mol})$ in $\mathrm{CH}_{2} \mathrm{Cl}_{2}$ $(5 \mathrm{~mL})$ at $0{ }^{\circ} \mathrm{C}$ was added $1,3,5$-trimethoxybenzene $(0.0286 \mathrm{~g}$, $0.170 \mathrm{mmol})$ and $\mathrm{BF}_{3} \cdot \mathrm{OEt}_{2}(17 \mu \mathrm{L}, 0.14 \mathrm{mmol})$. The solution was stirred for $0.5 \mathrm{~h}$ at $0{ }^{\circ} \mathrm{C}$, followed by $1.5 \mathrm{~h}$ at room temperature. Then, $\mathrm{NH}_{4} \mathrm{Cl}$ (saturated aq, $0.25 \mathrm{~mL}$ ) and $\mathrm{NaHCO}_{3}$ (saturated aq, $0.25 \mathrm{~mL}$ ) were added, followed by a conventional extractive workup $\left(\mathrm{CH}_{2} \mathrm{Cl}_{2}\right)$. Purification by preparative TLC
(2:1 hexanes/EtOAc, 2 developments) afforded $\mathbf{6 b}(0.0218 \mathrm{~g}$, $26.9 \mu \mathrm{mol}, 89 \%)$ as a red-brown oil. ${ }^{1} \mathrm{H}$ NMR $(500 \mathrm{MHz}$, $\left.\mathrm{CDCl}_{3}\right) \delta 8.95$ (br s, $\left.1 \mathrm{H}\right), 7.74(\mathrm{~s}, 1 \mathrm{H}), 6.27$ (dd, $J=8.8,5.4 \mathrm{~Hz}$, $1 \mathrm{H}), 6.14$ (s, 2H), 5.22 (d, $J=6.5 \mathrm{~Hz}, 1 \mathrm{H}), 4.36$ (dd, $J=11.6$, $4.9 \mathrm{~Hz}, 1 \mathrm{H}), 4.29(\mathrm{~m}, 1 \mathrm{H}), 4.27(\mathrm{~s}, 2 \mathrm{H}), 4.25(\mathrm{dd}, J=11.6$, $3.7 \mathrm{~Hz}, 1 \mathrm{H}), 3.82(\mathrm{~s}, 3 \mathrm{H}), 3.77$ (s, 6H), 2.60 (ddd, $J=14.2,5.3$, $1.3 \mathrm{~Hz}, 1 \mathrm{H}), 2.15(\mathrm{~m}, 1 \mathrm{H}), 2.13(\mathrm{~s}, 3 \mathrm{H}), 2.07$ (s, 3H); ${ }^{13} \mathrm{C}$ NMR $\left(125 \mathrm{MHz}, \mathrm{CDCl}_{3}\right) \delta 199.4,170.3,160.3,160.1,158.9,149.6$, 136.2, 115.1, 108.7, 104.1, 90.0, 85.7, 82.3, 81.1, 74.1, 63.8, 55.3, 54.7, 37.7, 26.5, 20.9, 20.6; IR (neat, ATR) 3200, 2997, 2962, 2088, 2048, 2018, 1746, 1711, 1664, $1598 \mathrm{~cm}^{-1}$; $\mathrm{ESI}^{+}-\mathrm{MS}(\mathrm{m} / \mathrm{z}):[\mathrm{M}]^{+}$calcd for $\mathrm{C}_{31} \mathrm{H}_{28} \mathrm{Co}_{2} \mathrm{~N}_{2} \mathrm{O}_{16}, 825.0000$; found, 825.0002 .

Hexacarbonyl dicobalt 3',5'-di- $O$-acetyl-2'-deoxy-5-(5-oxo-5phenylhex-1-yn-1-yl)uridine (6c). To a solution of nucleoside complex $4(0.0212 \mathrm{~g}, 30.6 \mu \mathrm{mol})$ in $\mathrm{CH}_{2} \mathrm{Cl}_{2}(5 \mathrm{~mL})$ at $0{ }^{\circ} \mathrm{C}$ was added acetophenone trimethylsilyl enol ether (trimethyl(1phenylvinyloxy)silane, $0.039 \mathrm{~g}, 0.20 \mathrm{mmol}$ ) and $\mathrm{BF}_{3} \cdot \mathrm{OEt}_{2}$ (16 $\mu \mathrm{L}, 0.13 \mathrm{mmol})$. The solution was stirred over $12 \mathrm{~h}$ with gradual warming to room temperature. Then, $\mathrm{NH}_{4} \mathrm{Cl}$ (saturated saturated aq, 5 drops) and $\mathrm{NaHCO}_{3}$ (saturated aq, 5 drops) were added, followed by a conventional extractive workup $\left(\mathrm{CH}_{2} \mathrm{Cl}_{2}\right)$. Purification by preparative TLC (3:2 hexanes/EtOAc, 2 developments) afforded $\mathbf{6 c}$ as brown oil (0.0108 g, $15.0 \mu \mathrm{mol}, 49 \%)$. ${ }^{1} \mathrm{H}$ NMR (500 MHz, $\left.\mathrm{CDCl}_{3}\right) \delta 8.68(\mathrm{~s}, 1 \mathrm{H}), 8.00(\mathrm{~d}, J=7.9 \mathrm{~Hz}$, $2 \mathrm{H}), 7.83(\mathrm{~s}, 1 \mathrm{H}), 7.58$ (t, $J=7.4 \mathrm{~Hz}, 1 \mathrm{H}), 7.48$ (apparent t, $J=$ $7.7 \mathrm{~Hz}, 2 \mathrm{H}), 6.26(\mathrm{dd}, J=8.7,5.4 \mathrm{~Hz}, 1 \mathrm{H}), 5.23$ (d, $J=6.4 \mathrm{~Hz}$, $1 \mathrm{H}), 4.39(\mathrm{dd}, J=11.7,5.4 \mathrm{~Hz}, 1 \mathrm{H}), 4.31(\mathrm{~m}, 1 \mathrm{H}), 4.26(\mathrm{dd}, J=$ 11.7, $3.8 \mathrm{~Hz}, 1 \mathrm{H}), 3.52(\mathrm{~m}, 2 \mathrm{H}), 3.42(\mathrm{t}, J=7.1 \mathrm{~Hz}, 2 \mathrm{H}), 2.71$ $(\mathrm{dd}, J=13.7,4.8 \mathrm{~Hz}, 1 \mathrm{H}), 2.19(\mathrm{~m}, 1 \mathrm{H}), 2.13(\mathrm{~s}, 3 \mathrm{H}), 2.08(\mathrm{~s}$, $3 \mathrm{H}) ;{ }^{13} \mathrm{C} \mathrm{NMR}\left(125 \mathrm{MHz}, \mathrm{CDCl}_{3}\right) \delta 199.2,198.3,170.29$, 170.26, 159.9, 149.3, 137.5, 136.6, 133.2, 128.7, 128.0, 114.1, $102.4,85.6,82.5,81.8,74.0,63.7,40.2,37.8,28.9,20.9,20.6$; IR (neat, ATR) 3208, 2956, 2926, 2089, 2050, 2018, 1746, 1715, 1688, $1597 \mathrm{~cm}^{-1}$; ESI ${ }^{+}-\mathrm{MS}(\mathrm{m} / \mathrm{z}):[\mathrm{M}+\mathrm{Na}]^{+}$calcd for $\mathrm{C}_{30} \mathrm{H}_{25} \mathrm{Co}_{2} \mathrm{~N}_{2} \mathrm{NaO}_{14}, 776.9789$; found, 776.9788.

Hexacarbonyl dicobalt 2',3',5'-tri- $O$-acetyl-5-(hex-5-en-1yn-1-yl)uridine (7a). To a solution of nucleoside complex 5 (20.6 mg, $28.4 \mu \mathrm{mol})$ and allyltrimethylsilane $(100 \mu \mathrm{L}$, $0.629 \mathrm{mmol})$ at $0{ }^{\circ} \mathrm{C}$ was added $\mathrm{SnCl}_{4}(90 \mu \mathrm{L}, 1.0 \mathrm{M}$, $0.090 \mathrm{mmol}$ ). The solution was stirred for $1 \mathrm{~h}$ at $0{ }^{\circ} \mathrm{C}$, followed by $2 \mathrm{~h}$ at rt. Then, $\mathrm{NH}_{4} \mathrm{Cl}$ (saturated aq, 5 drops) and $\mathrm{NaHCO}_{3}$ (saturated aq, 5 drops) were added, and a conventional extractive workup was performed $\left(\mathrm{CH}_{2} \mathrm{Cl}_{2}\right)$. Preparative TLC (3:2 petroleum ether/EtOAc) afforded, in order of elution, 7a (7.8 $\mathrm{mg}, 11 \mu \mathrm{mol}, 37 \%$ yield, 46\% brsm) and recovered 5 (3.7 mg, $5.1 \mu \mathrm{mol}, 18 \%$ recovery). 7a: ${ }^{1} \mathrm{H}$ NMR $(300 \mathrm{MHz}$, $\left.\mathrm{CDCl}_{3}\right) \delta 8.60(\mathrm{~s}, 1 \mathrm{H}), 7.55(\mathrm{~s}, 1 \mathrm{H}), 6.00(\mathrm{~d}, J=5.4 \mathrm{~Hz}, 1 \mathrm{H})$, $5.94(\mathrm{~m}, 1 \mathrm{H}), 5.30-5.40(\mathrm{~m}, 2 \mathrm{H}), 5.14(\mathrm{~d}, J=17.1 \mathrm{~Hz}, 1 \mathrm{H})$, 
$5.06(\mathrm{~d}, J=10.2 \mathrm{~Hz}, 1 \mathrm{H}), 4.23-4.43(\mathrm{~m}, 3 \mathrm{H}), 3.17$ (apparent t, $J=7.9 \mathrm{~Hz}, 2 \mathrm{H}), 2.43(\mathrm{~m}, 2 \mathrm{H}), 2.16(\mathrm{~s}, 3 \mathrm{H}), 2.13(\mathrm{~s}, 3 \mathrm{H}), 2.10$ $(\mathrm{s}, 3 \mathrm{H}) ;{ }^{13} \mathrm{C} \mathrm{NMR}\left(125 \mathrm{MHz}, \mathrm{CDCl}_{3}\right) \delta 199.2,170.3,169.5$, 159.6, 149.4, 137.9, 137.2, 115.7, 115.2, 103.6, 88.0, 80.3, 80.1, $72.4,70.3,63.3,35.6,33.8,20.6,20.5,20.4$; IR (neat, ATR) 3219, 2956, 2924, 2089, 2049, 2014, 1749, 1718, $1692 \mathrm{~cm}^{-1}$; $\mathrm{ESI}^{+}-\mathrm{MS}(\mathrm{m} / \mathrm{z}):[\mathrm{M}+\mathrm{Na}]^{+}$calcd for $\mathrm{C}_{27} \mathrm{H}_{24} \mathrm{Co}_{2} \mathrm{~N}_{2} \mathrm{NaO}_{15}$, 756.9738; found, 756.9742 .

Hexacarbonyl dicobalt 2',3',5'-tri- $O$-acetyl-5-[3-(2,4,6trimethoxyphenyl)prop-1-yn-1-yl)]uridine (7b). To a solution of nucleoside complex $5(20.4 \mathrm{mg}, 28.2 \mu \mathrm{mol})$ and 1,3,5trimethoxybenzene $(23.6 \mathrm{mg}, 140 \mu \mathrm{mol})$ in $\mathrm{CH}_{2} \mathrm{Cl}_{2}(5 \mathrm{~mL})$ at $0{ }^{\circ} \mathrm{C}$ was added $\mathrm{BF}_{3} \cdot \mathrm{OEt}_{2}(11 \mu \mathrm{L}, 87 \mu \mathrm{mol})$. The solution was stirred for $45 \mathrm{~min}$ at $0{ }^{\circ} \mathrm{C}$, followed by $1 \mathrm{~h}$ at rt. Then, $\mathrm{NH}_{4} \mathrm{Cl}$ (saturated aq, 5 drops) and $\mathrm{NaHCO}_{3}$ (saturated aq, 5 drops) were added, and a conventional extractive workup was performed $\left(\mathrm{CH}_{2} \mathrm{Cl}_{2}\right)$. Preparative TLC (3:2 hexanes/EtOAc) gave 7b as viscous brown oil (11.3 mg, $13.2 \mu \mathrm{mol}, 47 \%) .{ }^{1} \mathrm{H}$ NMR $\left(300 \mathrm{MHz}, \mathrm{CDCl}_{3}\right) \delta 8.64(\mathrm{~s}, 1 \mathrm{H}), 7.56(\mathrm{~s}, 1 \mathrm{H}), 6.14(\mathrm{~s}, 2 \mathrm{H})$, $5.92(\mathrm{~d}, J=5.7 \mathrm{~Hz}, 1 \mathrm{H}), 5.32-5.42(\mathrm{~m}, 2 \mathrm{H}), 4.20-4.45(\mathrm{~m}, 5 \mathrm{H})$, $3.82(\mathrm{~s}, 3 \mathrm{H}), 3.78(\mathrm{~s}, 6 \mathrm{H}), 2.16(\mathrm{~s}, 3 \mathrm{H}), 2.12(\mathrm{~s}, 3 \mathrm{H}), 2.11(\mathrm{~s}$, $3 \mathrm{H}) ;{ }^{13} \mathrm{C} \mathrm{NMR}\left(125 \mathrm{MHz}, \mathrm{CDCl}_{3}\right) \delta 199.3,170.3,169.5,160.4$, 159.9, 158.9, 149.6, 136.7, 115.6, 108.7, 103.9, 90.0, 87.9, 80.5, $80.1,72.4,70.3,63.3,55.3,54.8,25.6,20.6,20.5,20.3$; IR (neat, ATR) 3211, 2956, 2924, 2087, 2047, 2010, 1748, 1716, 1693, $1597 \mathrm{~cm}^{-1}$; $\mathrm{ESI}^{+}-\mathrm{MS}(\mathrm{m} / \mathrm{z}):[\mathrm{M}+\mathrm{Na}]^{+}$calcd for $\mathrm{C}_{33} \mathrm{H}_{30} \mathrm{Co}_{2} \mathrm{~N}_{2} \mathrm{NaO}_{18}$, 883.0055; found, 883.0077.

Hexacarbonyl dicobalt 2',3',5'-tri-O-acetyl-5-[3-(1'methylindol-3'-yl)prop-1-yn-1-yl)]uridine (7d). To a solution of nucleoside complex $\mathbf{5}(20.4 \mathrm{mg}, 28.1 \mu \mathrm{mol})$ and $N$-methylindole $(18.4 \mathrm{mg}, 14.0 \mu \mathrm{mol})$ in $\mathrm{CH}_{2} \mathrm{Cl}_{2}(5 \mathrm{~mL})$ at $0{ }^{\circ} \mathrm{C}$ was added $\mathrm{BF}_{3} \cdot \mathrm{OEt}_{2}(14 \mu \mathrm{L}, 0.11 \mathrm{mmol})$. The solution was stirred for $45 \mathrm{~min}$ at $0{ }^{\circ} \mathrm{C}$, followed by $\mathrm{rt}$ for $45 \mathrm{~min}$. Then, $\mathrm{NH}_{4} \mathrm{Cl}$ (saturated aq, 5 drops) and $\mathrm{NaHCO}_{3}$ (saturated aq, 5 drops) were added, and a conventional extractive workup was performed $\left(\mathrm{CH}_{2} \mathrm{Cl}_{2}\right)$. Preparative TLC (3:2 hexanes/EtOAc) afforded $\mathbf{7 d}$ as brown oil (9.2 mg, $11 \mu \mathrm{mol}, 40 \%) .{ }^{1} \mathrm{H}$ NMR $(300 \mathrm{MHz}$, $\left.\mathrm{CDCl}_{3}\right) \delta 8.39($ br s, $1 \mathrm{H}), 7.63(\mathrm{~d}, J=7.8 \mathrm{~Hz}, 1 \mathrm{H}), 7.46(\mathrm{~s}, 1 \mathrm{H})$, 7.29 (d, obscured, 1H), 7.24 (apparent t, $J=7.0 \mathrm{~Hz}, 1 \mathrm{H}$ ), 7.12 (apparent dt, $J=1.0,7.4 \mathrm{~Hz}, 1 \mathrm{H}), 6.96(\mathrm{~s}, 1 \mathrm{H}), 5.84(\mathrm{~d}, J=$ $5.4 \mathrm{~Hz}, 1 \mathrm{H}), 5.28-5.38(\mathrm{~m}, 2 \mathrm{H}), 4.52(\mathrm{~s}, 2 \mathrm{H}), 4.23-4.40(\mathrm{~m}$ $3 \mathrm{H}), 3.79(\mathrm{~s}, 3 \mathrm{H}), 2.15(\mathrm{~s}, 3 \mathrm{H}), 2.11(\mathrm{~s}, 3 \mathrm{H}), 2.09$ (s, 3H); ${ }^{13} \mathrm{C}$ NMR $\left(125 \mathrm{MHz}, \mathrm{CDCl}_{3}\right) \delta$ 199.1, 170.3, 169.53, 169.52, 158.9, 149.3, 138.1, 136.6, 127.7, 127.6, 121.6, 119.0, 118.7, 115.3, 113.8, 109.2, 106.6, 88.5, 81.3, 80.0, 72.4, 70.2, 63.2, 32.7, 29.6, 20.6, 20.5, 20.4; IR (neat, ATR) 3204, 2954, 2924, 2089, 2050, 2019, 1750, 1720, $1692 \mathrm{~cm}^{-1}$; ESI ${ }^{+}-\mathrm{MS}(\mathrm{m} / \mathrm{z})$ : $[\mathrm{M}+\mathrm{H}]^{+}$calcd for $\mathrm{C}_{33} \mathrm{H}_{27} \mathrm{Co}_{2} \mathrm{~N}_{3} \mathrm{O}_{15}, 823.0184$; found, 823.0184 .

\section{Supporting Information}

\section{Supporting Information File 1}

${ }^{1} \mathrm{H}$ and ${ }^{13} \mathrm{C}$ NMR spectra for compounds $2,3,4,5, \mathbf{6 a}-\mathbf{c}$, and $\mathbf{7 a}, \mathbf{b}, \mathbf{d}$.

[https://www.beilstein-journals.org/bjoc/content/

supplementary/1860-5397-16-1-S1.pdf]

\section{Acknowledgements}

We thank the National Institutes of Health (NIH, CA111329), the Statutory Funds of CMMS PAS, and the Natural Sciences and Engineering Research Council (NSERC Canada, RG-PIN2016-04946) for support of this research. The NSF awards (CHE-0821487, CHE-1048719) and OU Research Excellence Fund are also acknowledged. We are also thankful to Dr. Hiroyuki Hayakawa (Yamasa Corporation, Biochemicals Division) for a generous supply of nucleosides.

\section{ORCID ${ }^{\circledR}$ iDs}

Renata Kaczmarek - https://orcid.org/0000-0002-3869-1068

James R. Green - https://orcid.org/0000-0003-2334-4503

\section{References}

1. Herdewijn, P. Modified Nucleosides in Biochemistry, Biotechnology and Medicine; Wiley-VCH: Weinheim, Germany, 2008. doi:10.1002/9783527623112

2. Godefridus, J. P., Ed. Deoxynucleoside Analogs in Cancer Therapy; Humana Press: Totowa, USA, 2006.

3. Merino, P., Ed. Chemical Synthesis of Nucleoside Analogues; John Wiley \& Sons: Hoboken, NJ, USA, 2013. doi:10.1002/9781118498088

4. Jordheim, L. P.; Durantel, D.; Zoulim, F.; Dumontet, C. Nat. Rev. Drug Discovery 2013, 12, 447-464. doi:10.1038/nrd4010

5. Bobrovnikova-Marjon, E.; Hurov, J. B. Annu. Rev. Med. 2014, 65, 157-170. doi:10.1146/annurev-med-092012-112344

6. Kore, A. R.; Charles, I. Curr. Org. Chem. 2012, 16, 1996-2013. doi:10.2174/138527212803251622

7. Kapdi, A. R.; Maiti, D.; Sanghvi, Y. S., Eds. Palladium-Catalyzed Modification of Nucleosides, Nucleotides and Oligonucleotides; Elsevier: Amsterdam, Netherlands, 2018. doi:10.1016/c2016-0-00656-3

8. Hilko, D. H.; Bornaghi, L. F.; Poulsen, S.-A. J. Org. Chem. 2018, 83, 11944-11955. doi:10.1021/acs.joc.8b01834 See for a recent representative example of C-5 modified uridines.

9. Bag, S. S.; Gogoi, H. J. Org. Chem. 2018, 83, 7606-7621. doi:10.1021/acs.joc.7b03097 See for a recent representative example of C-5 modified uridines. 10. Barthes, N. P. F.; Karpenko, I. A.; Dziuba, D.; Spadafora, M.; Auffret, J.; Demchenko, A. P.; Mély, Y.; Benhida, R.; Michel, B. Y.; Burger, A. RSC Adv. 2015, 5, 33536-33545. doi:10.1039/c5ra02709h See for a ecent representative example of C-5 modified uridines.

11. Hottin, A.; Marx, A. Acc. Chem. Res. 2016, 49, 418-427. doi:10.1021/acs.accounts.5b00544

12. Mei, H.; Chaput, J. C. Chem. Commun. 2018, 54, 1237-1240. doi:10.1039/c7cc09130c 
13. Sniady, A.; Durham, A.; Morreale, M. S.; Marcinek, A.; Szafert, S.; Lis, T.; Brzezinska, K. R.; Iwasaki, T.; Ohshima, T.; Mashima, K.; Dembinski, R. J. Org. Chem. 2008, 73, 5881-5889.

doi:10.1021/j08007995

and references cited therein.

14. Rao, M. S.; Esho, N.; Sergeant, C.; Dembinski, R. J. Org. Chem. 2003, 68, 6788-6790. doi:10.1021/j00345648

15. Sniady, A.; Sevilla, M. D.; Meneni, S.; Lis, T.; Szafert, S.; Khanduri, D.; Finke, J. M.; Dembinski, R. Chem. - Eur. J. 2009, 15, 7569-7577. doi:10.1002/chem.200900481

16. Franz, K. J.; Metzler-Nolte, N., Eds. Metals in Medicine. Chem. Rev. 2019, 119, 727-1624. doi:10.1021/acs.chemrev.8b00685

17. Jaouen, G.; Salmain, M., Eds. Bioorganometallic Chemistry: Applications in Drug Discovery, Biocatalysis, and Imaging; John Wiley \& Sons: Hoboken, NJ, USA, 2015.

18. Kraatz, H. B.; Metzler-Nolte, N., Eds. Concepts and Models in Bioinorganic Chemistry; Wiley-VCH: Weinheim, Germany, 2006.

19. Jaouen, G., Ed. Bioorganometallic Chemistry; Wiley-VCH: Weinheim, Germany, 2006.

20. Gasser, G. Chimia 2015, 69, 442-446. doi:10.2533/chimia.2015.442

21. Gasser, G.; Ott, I.; Metzler-Nolte, N. J. Med. Chem. 2011, 54, 3-25. doi:10.1021/jm100020w

22. Timerbaev, A. R.; Hartinger, C. G.; Aleksenko, S. S.; Keppler, B. K. Chem. Rev. 2006, 106, 2224-2248. doi:10.1021/cr040704h

23. Hartinger, C. G.; Metzler-Nolte, N.; Dyson, P. J. Organometallics 2012, 31, 5677-5685. doi:10.1021/om300373t

24. Ott, I.; Gust, R. Arch. Pharm. (Weinheim, Ger.) 2007, 340, 117-126. doi:10.1002/ardp.200600151

25. Heffern, M. C.; Yamamoto, N.; Holbrook, R. J.; Eckermann, A. L.; Meade, T. J. Curr. Opin. Chem. Biol. 2013, 17, 189-196. doi:10.1016/j.cbpa.2012.11.019

26. Li, J.; Zhang, J.; Zhang, Q.; Wang, Y.; Bai, Z.; Zhao, Q.; He, D.; Wang, Z.; Zhang, J.; Chen, Y. Bioorg. Med. Chem. 2019, 27, 115071. doi:10.1016/j.bmc.2019.115071 See for a recent representative example.

27. Berrino, E.; Milazzo, L.; Micheli, L.; Vullo, D.; Angeli, A.; Bozdag, M.; Nocentini, A.; Menicatti, M.; Bartolucci, G.; di Cesare Mannelli, L.; Ghelardini, C.; Supuran, C. T.; Carta, F. J. Med. Chem. 2019, 62, 7233-7249. doi:10.1021/acs.jmedchem.9b00845

28. Atkin, A. J.; Williams, S.; Sawle, P.; Motterlini, R.; Lynam, J. M.; Fairlamb, I. J. S. Dalton Trans. 2009, 3653-3656. doi:10.1039/b904627p

29. Ott, I.; Kircher, B.; Dembinski, R.; Gust, R. Expert Opin. Ther. Pat. 2008, 18, 327-337. doi:10.1517/13543776.18.3.327

30. Munteanu, C. R.; Suntharalingam, K. Dalton Trans. 2015, 44, 13796-13808. doi:10.1039/c5dt02101d

31. Kaczmarek, R.; Korczyński, D.; Królewska-Golińska, K.; Wheeler, K. A.; Chavez, F. A.; Mikus, A.; Dembinski, R. ChemistryOpen 2018, 7, 237-247. doi:10.1002/open.201700168 and references cited therein.

32. Sergeant, C. D.; Ott, I.; Sniady, A.; Meneni, S.; Gust, R.; Rheingold, A. L.; Dembinski, R. Org. Biomol. Chem. 2008, 6, 73-80. doi:10.1039/b713371e

33. Meneni, S.; Ott, I.; Sergeant, C. D.; Sniady, A.; Gust, R.; Dembinski, R. Bioorg. Med. Chem. 2007, 15, 3082-3088.

doi:10.1016/j.bmc.2007.01.048

See for anticancer activity of precursors 5-alkynyl 2'-deoxyuridines.

34. Kann, N. Curr. Org. Chem. 2012, 16, 322-334. doi:10.2174/138527212799499949
35. Shea, K. M. Nicholas Reaction. In Name Reactions for Homologations, Part 1; Li, J. J., Ed.; John Wiley \& Sons: Hoboken, NJ, USA, 2009; pp 284-298.

36. Díaz, D. D.; Betancort, J. M.; Martín, V. S. Synlett 2007, 343-359. doi:10.1055/s-2007-967958

37. Teobald, B. J. Tetrahedron 2002, 58, 4133-4170. doi:10.1016/s0040-4020(02)00315-0

38. Green, J. R. Curr. Org. Chem. 2001, 5, 809-826. doi:10.2174/1385272013375247

39. Caffyn, A. J. M.; Nicholas, K. M. Transition Metal Alkyne Complexes: Transition Metal-stabilized Propargyl Systems. In Comprehensive Organometallic Chemistry II; Abel, E. W.; Stone, F. G. A.; Wilkinson, G., Eds.; Pergamon: Oxford, U.K., 1995; Vol. 12, pp 685-702. doi:10.1016/b978-008046519-7.00124-6

40. Green, J. R.; Nicholas, K. M. Org. React., in press.

41. Roth, K.-D. Synlett 1993, 529-533. doi:10.1055/s-1993-22518

42. Taj, R. A.; Green, J. R. J. Org. Chem. 2010, 75, 8258-8270. doi:10.1021/j0102127q

43. Krafft, M. E.; Cheung, Y. Y.; Wright, C.; Cali, R. J. Org. Chem. 1996, 61, 3912-3915. doi:10.1021/j0952160c

44. O'Boyle, J. E.; Nicholas, K. M. Tetrahedron Lett. 1980, 21, 1595-1598. doi:10.1016/s0040-4039(00)77762-8

45. Takano, S.; Sugihara, T.; Ogasawara, K. Synlett 1992, 70-72. doi:10.1055/s-1992-21270

46. Green, J. R. Chem. Commun. 1998, 1751-1752. doi:10.1039/a803316a

47. Nicholas, K. M.; Mulvaney, M.; Bayer, M. J. Am. Chem. Soc. 1980, 102, 2508-2510. doi:10.1021/ja00527a086

48. Tanino, K.; Shimizu, T.; Miyama, M.; Kuwajima, I. J. Am. Chem. Soc. 2000, 122, 6116-6117. doi:10.1021/ja001003e

49. St Onge, B.; Green, J. R. Synlett 2017, 28, 2923-2927. doi:10.1055/s-0036-1588528

50. Green, J. R. Eur. J. Org. Chem. 2008, 6053-6062. doi:10.1002/ejoc.200800836

51. Isobe, M.; Hamajima, A. Nat. Prod. Rep. 2010, 27, 1204-1226. doi:10.1039/b919467n

52. Hernandez, J. N.; Ramirez, M. A.; Rodriguez, M. L.; Martin, V. S. Org. Lett. 2008, 10, 2349-2352. doi:10.1021/ol800544a

53. Miyazaki, A.; Asanuma, M.; Dodo, K.; Egami, H.; Sodeoka, M. Chem. - Eur. J. 2014, 20, 8116-8128. doi:10.1002/chem.201400056

54. Prasad, J. S.; Liebeskind, L. S. Tetrahedron Lett. 1987, 28 , 1857-1860. doi:10.1016/s0040-4039(00)95993-8

55. Gruselle, M.; Cordier, C.; Salmain, M.; El Amouri, H.; Guerin, C.; Vaissermann, J.; Jaouen, G. Organometallics 1990, 9, 2993-2997. doi:10.1021/om00161a031 See for a representative example.

56. Tanaka, S.; Isobe, M. Tetrahedron 1994, 50, 5633-5644. doi:10.1016/s0040-4020(01)85634-9

57. Jiang, Y.; Isobe, M. Tetrahedron 1996, 52, 2877-2892. doi:10.1016/0040-4020(96)00008-7

58. Mukai, C.; Itoh, T.; Hanaoka, M. Tetrahedron Lett. 1997, 38 , 4595-4598. doi:10.1016/s0040-4039(97)00983-0

59. Hosokawa, S.; Isobe, M. J. Org. Chem. 1999, 64, 37-48. doi:10.1021/jo980088n

60. Gómez, A. M.; Uriel, C.; Valverde, S.; López, J. C. Org. Lett. 2006, 8, 3187-3190. doi:10.1021/ol060929+

61. Lobo, F.; Gómez, A. M.; Miranda, S.; López, J. C. Chem. - Eur. J. 2014, 20, 10492-10502. doi:10.1002/chem.201402149

62. Bag, S. S.; Das, S. K. Tetrahedron 2019, 75, 3024-3037. doi:10.1016/j.tet.2019.04.038 
63. Esho, N.; Davies, B.; Lee, J.; Dembinski, R. Chem. Commun. 2002, 332-333. doi:10.1039/b109501c

64. Yamamoto, Y.; Seko, T.; Nakamura, H.; Nemoto, H. Heteroat. Chem 1992, 3, 239-244. doi:10.1002/hc.520030308

To our knowledge, compounds 2 and 3 have not yet been reported.

See for a related protected compound.

65. Tolstikov, V. V.; Stetsenko, D. A.; Potapov, V. K.; Sverdlov, E. D.

Nucleosides Nucleotides 1997, 16, 215-225.

doi:10.1080/07328319708001343

See for a related protected compound.

\section{License and Terms}

This is an Open Access article under the terms of the Creative Commons Attribution License

(https://creativecommons.org/licenses/by/4.0). Please note that the reuse, redistribution and reproduction in particular requires that the authors and source are credited.

The license is subject to the Beilstein Journal of Organic

Chemistry terms and conditions:

(https://www.beilstein-journals.org/bjoc)

The definitive version of this article is the electronic one which can be found at:

doi:10.3762/bjoc. 16.1 